\title{
Effects of a standard hyperbaric oxygen treatment protocol on pulmonary function
}

\author{
E. Thorsen, L. Aanderud, T.B. Aasen
}

\begin{abstract}
Effects of a standard hyperbaric oxygen treatment protocol on pulmonary function. E. Thorsen, L. Aanderud, T.B. Aasen. @ERS Journals Ltd 1998.

ABSTRACT: The prescription of hyperbaric oxygen (HBO) therapy for disorders not related to diving is increasing. Pulmonary oxygen toxicity is well known, but the effect of the cumulative oxygen exposure corresponding to a standard HBO treatment protocol has not been quantified before.

Twenty patients (10 male) had 21 HBO treatments at a partial pressure of oxygen of $240 \mathrm{kPa}$ for $90 \mathrm{~min}$ daily. None had any previous lung disease and all had normal chest radiography and lung function at the start of the study. Dynamic lung volumes, forced expiratory flows and the transfer factor of the lung for carbon monoxide ( $T \mathrm{~L}, \mathrm{CO})$ were measured before the $\mathrm{HBO}$ treatment, on days 7, 14 and 21 during treatment and then 3-4 weeks after treatment.

Four patients (one male) reported nonproductive coughing during the last week of treatment. There was a progressive reduction in forced expiratory volume in one second (FEV1) $(\mathbf{p}<0.001)$, mean forced mid-expiratory flow rate $(\mathrm{FEF} 25-75 \%)(\mathbf{p}<0.001)$ and forced expiratory flows at 50 and $75 \%$ of forced vital capacity (FVC) expired during HBO treatment. The reduction in FEV1 on day 21 was $4.4 \pm 1.7 \%$ and in FEF 25-75\% 10.3 $\pm 6.1 \%$. Four weeks after treatment there was a partial normalization. There were no changes in FVC or peak expiratory flow (PEF). TL,CO was slightly reduced on day 21 of treatment only $(p<0.01)$ and fully normalized 1 month later.

A reduction in small airways conductance is consistent with other studies where total oxygen exposures have been below the limit causing toxic pulmonary effects traditionally measured as a reduction in vital capacity. This effect is not considered to be of any clinical significance for patients treated with hyperbaric oxygen unless repeated treatment series are to be given.

Eur Respir J 1998; 12: 1442-1445.
\end{abstract}

Depts of Hyperbaric and Occupational Medicine, Haukeland Hospital, Bergen, Norway.

Correspondence: E. Thorsen

Dept of Hyperbaric Medicine

Haukeland Hospital

N-5021 Bergen

Norway

Fax: 4755975137

Keywords: Diving hyperbaric oxygen oxygen toxicity

pulmonary function

Received: January 191998

Accepted after revision July 141998
Recompression and hyperbaric oxygen (HBO) are used in the treatment for diving-related diseases such as decompression sickness and arterial gas embolism. For a long time HBO has also been shown to be effective in carbon monoxide poisoning and anaerobic infections. More recently, HBO has been shown to have supplementary effects in the treatment of other disorders characterized by local ischaemia. An increase in local oxygen supply due to an increased gradient for diffusion is achieved by increasing the partial pressure of oxygen $\left(\mathrm{PO}_{2}\right)$ in inspired gas. This results in local stimulation of fibroblast proliferation and collagen synthesis, angiogenesis and enhanced granulocyte function and peroxidase activity in ischaemic tissue. In this way, HBO treatment is an effective adjunct in the treatment of osteoradionecrosis, chronic osteomyelitis, diabetic leg ulcers and radiation-induced proctitis and cystitis. On an experimental basis, HBO treatment is currently evaluated as a supplement in the treatment of several other disorders. Indications for HBO treatment have been worked out by the Undersea and Hyperbaric Society [1], differentiating between indications where $\mathrm{HBO}$ has been shown to have a definite effect based on controlled clinical studies and indications where HBO still has to be considered experimental. In this setting, $\mathrm{HBO}$ treatment is usually given for 90 min daily at a $\mathrm{PO}_{2}$ of $200-280 \mathrm{kPa}$ for 20-30 days.
Toxic pulmonary effects of exposure to hyperoxia are well known. There is a dose-dependent reduction in vital capacity with continuous exposure to a $\mathrm{PO}_{2}>50 \mathrm{kPa}$, as characterized by CLARK and LAMBERTSEN [2]. It has also been shown that this effect is attenuated by intermittent exposure to up to the same cumulative dose of oxygen [3] and tolerance to the oxidative stress develops. HBO treatment protocols and diving procedures are based on these doseresponse relationships and practical experience. Ser-ious pulmonary oxygen toxicity has not been reported with this form of HBO treatment. However, systematic studies to quantify the effect on pulmonary function of commonly used HBO treatment protocols are lacking.

\section{Methods}

\section{Patients}

Twenty consecutive patients (10 male) undergoing treatment for ischaemic leg or foot ulcers, chronic osteomyelitis, delayed healing of fractures with pseudarthrosis or pelvic radionecrosis were included in the study. Patients with lung disease, former irradiation of the head, neck or thorax as part of the treatment for the primary disease, current smokers and patients with radiologically abnormal 
Table 1. - Subject characteristics and pulmonary function before hyperbaric oxygen treatment

\begin{tabular}{|c|c|c|}
\hline & Males & Females \\
\hline Age yrs & $54 \pm 18$ & $46 \pm 12$ \\
\hline Height $\mathrm{cm}$ & $181 \pm 8$ & $162 \pm 6$ \\
\hline Body mass $\mathrm{kg}$ & $79 \pm 13$ & $58 \pm 8$ \\
\hline FVC L & $4.96 \pm 0.82$ & $3.67 \pm 0.47$ \\
\hline$\%$ pred & $103 \pm 7$ & $109 \pm 10$ \\
\hline FEV1 L & $3.71 \pm 0.65$ & $2.93 \pm 0.61$ \\
\hline$\%$ pred & $99 \pm 7$ & $104 \pm 9$ \\
\hline$T \mathrm{~L}, \mathrm{CO} \quad \mathrm{mmol} \cdot \mathrm{min}^{-1} \cdot \mathrm{kPa}^{-1}$ & $9.72 \pm 1.19$ & $7.55 \pm 2.32$ \\
\hline$\%$ pred & $97 \pm 7$ & $95 \pm 17$ \\
\hline
\end{tabular}

FVC: forced vital capacity; \% pred: percentage of predicted value; FEV1: forced expiratory volume in one second; $T \mathrm{~L}, \mathrm{CO}$ : transfer factor of the lung for carbon monoxide.

findings in the lung parenchyma were excluded. Four had stopped smoking $>1$ yr before the study, six had stopped smoking in the last year before the study and 10 were never-smokers. Their age, height, body mass and pulmonary function at the start of the study are given in table 1 .

\section{Hyperbaric oxygen treatment}

HBO treatment was given daily in the morning on 21 consecutive days. The patients were compressed in a hyperbaric chamber to a pressure of $240 \mathrm{kPa}$ within $10-15$ min. They were then given oxygen by an oronasal mask at a pressure of $240 \mathrm{kPa}$ in three cycles of $30 \mathrm{~min}$, interrupted by breathing air at a pressure of $240 \mathrm{kPa}$ for two periods of $5 \mathrm{~min}$ in-between. Thereafter, they were decompressed to normal atmospheric pressure in 7-10 min. The outline of the daily oxygen exposure is given in figure 1 . The HBO treatment was given in the hyperbaric chamber complex at the Norwegian Underwater Technology Centre.

\section{Lung function measurements}

Measurements were taken in the morning before entering the hyperbaric chamber on days $1,7,14$ and 21 of treatment. A control measurement was taken 3-4 weeks

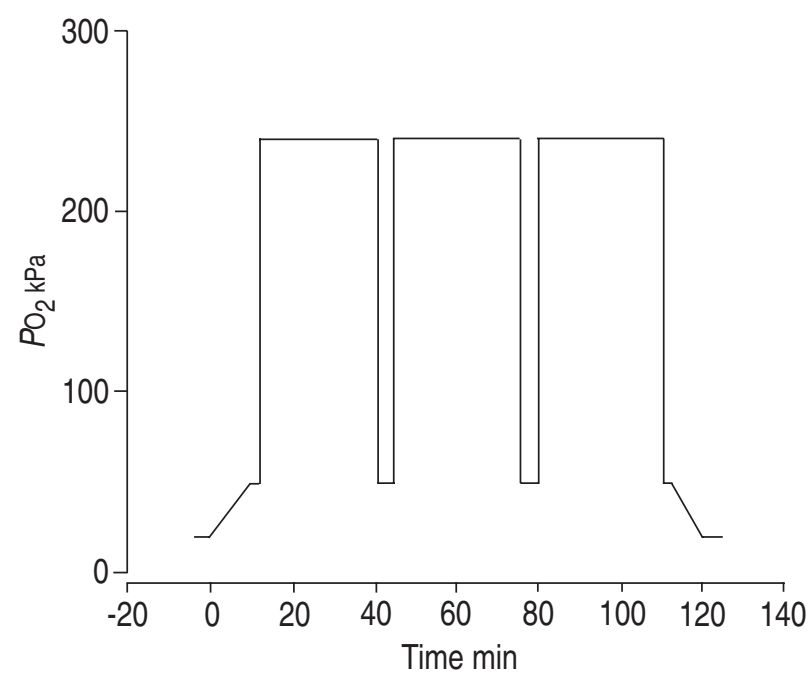

Fig. 1. - Daily oxygen exposure of patients having hyperbaric oxygen treatment for 21 days. Three cycles of oxygen of $30 \mathrm{~min}$ at an oxygen tension $\left(\mathrm{PO}_{2}\right)$ of $240 \mathrm{kPa}$ are given, interrupted by two periods breathing air at a total pressure of $240 \mathrm{kPa}\left(\mathrm{PO}_{2}=50 \mathrm{kPa}\right)$. thereafter. Measurements of lung function included forced expiratory lung volumes, forced expiratory flows and the transfer factor of the lung for carbon monoxide (TL,CO). The measurements were made on a Morgan Benchmark lung function testing apparatus (PK Morgan, Kent, UK). All measurements were taken by the same technician at the same time of the day.

The forced vital capacity (FVC), forced expiratory volume in one second (FEV1) and peak expiratory flow rate $(\mathrm{PEF})$ were taken as the highest readings obtained from at least three satisfactory forced expiratory manoeuvres. Mean forced mid-expiratory flow rate (FEF25-75\%) and forced expiratory flow rates at 25,50 and $75 \%$ of FVC expired (FEF25\%, FEF50\% and FEF75\%) were taken as the best values from flow-volume loops not differing by $>5 \%$ from the highest FVC. TL,CO was measured by the single breath-holding technique. Effective alveolar volume (VA) was measured simultaneously by helium dilution and the transfer coefficient for carbon monoxide $(K \mathrm{CO})$ was calculated as $T \mathrm{~L}, \mathrm{CO} / \mathrm{VA}$. An earlobe or fingertip blood sample was analysed for haemoglobin concentration on a Hemoglobin Photometer (Mecatronic AB, Helsingborg, Sweden) just before the lung function measurements. A correction in TL,CO to a haemoglobin concentration of 146 $\mathrm{g} \cdot \mathrm{L}^{-1}$ (TL,CO,corr) was made [4]. The better of the two measurements of $T \mathrm{~L}, \mathrm{CO}$ was used in the analysis

Measurements of lung function were made according to the standardized procedures of the European Respiratory Society (ERS) [5, 6]. Volume calibration of the spirometer and calibration of the gas analysers were done before each measurement. All measurements were corrected to a body temperature, pressure, saturated (BTPS) condition. At the start of HBO treatment, the patients' baseline lung function was compared with the reference values of the ERS for FVC, FEV1 and TL,CO [5, 6].

\section{Statistics}

Changes in the lung function variables were calculated as the percentage difference from the first measurement before HBO treatment on each follow-up examination. Differences from this baseline were tested using a paired t-test with the Bonferroni method of adjusting the level of significance. A p-value $<0.01$ was considered to be significant. Separate analyses in males and females were performed. Any relationship between changes in the lung function variables and age or baseline lung function was tested with simple correlation analysis. All results are given as mean $\pm \mathrm{SD}$.

\section{Results}

All patients, except for one previous smoker whose $T \mathrm{~L}, \mathrm{CO}$ was $58 \%$ of predicted, had lung function within the normal predicted range at the start of the study (table 1). Four patients (one male) reported slight nonproductive coughing during the last week of treatment, which subsided during the follow-up period.

There were no significant changes in FVC, FEF25\% or PEF (table 2). There were significant reductions in FEV1 $(\mathrm{p}<0.001), \mathrm{FEF} 25-75 \%,(\mathrm{p}<0.001)$, FEF50\% and FEF75\% $(\mathrm{p}<$ $0.01)$ at the end of the $\mathrm{HBO}$ treatment period. The mean reduction in FEV1 was $4.4 \pm 1.7 \%$ and in FEF25-75\% 10.3 \pm $6.1 \%$. The gradual fall in these parameters during the treatment period had stopped at the follow-up examination, but 
Table 2. - Changes in lung function during hyperbaric oxygen (HBO) treatment and at 4 weeks' follow-up in 10 male and 10 female patients

\begin{tabular}{|c|c|c|c|c|c|}
\hline & $\begin{array}{c}\text { Before } \mathrm{HBO} \\
\text { treatment }\end{array}$ & $\begin{array}{c}\% \text { change after } 7 \\
\text { days' treatment }\end{array}$ & $\begin{array}{c}\% \text { change after } 14 \\
\text { days' HBO treatment }\end{array}$ & $\begin{array}{c}\text { \% change after } 21 \\
\text { days' HBO treatment }\end{array}$ & $\begin{array}{l}\% \text { change } 4 \text { weeks } \\
\text { after end of HBO } \\
\text { treatment }\end{array}$ \\
\hline$\overline{\text { FVC L }}$ & $4.32 \pm 0.92$ & $-1.5 \pm 2.4$ & $-1.0 \pm 1.5$ & $-0.7 \pm 1.6$ & $-1.2 \pm 2.3$ \\
\hline FEV1 L & $3.32 \pm 0.74$ & $-2.0 \pm 2.4$ & $-3.2 \pm 1.9 * *$ & $-4.4 \pm 1.7 * * *$ & $-3.5 \pm 3.2 * *$ \\
\hline FEF $25-75 \% \mathrm{~L} \cdot \mathrm{s}^{-1}$ & $3.08 \pm 1.28$ & $-3.9 \pm 7.5$ & $-8.6 \pm 5.2 * *$ & $-10.3 \pm 6.1 * * *$ & $-10.7 \pm 8.4 * * *$ \\
\hline $\mathrm{FEF} 25 \% \mathrm{~L} \cdot \mathrm{S}^{-1}$ & $7.79 \pm 2.12$ & $-1.1 \pm 7.1$ & $-4.5 \pm 8.2$ & $-3.2 \pm 6.7$ & $3.0 \pm 7.0$ \\
\hline FEF50\% L $\cdot \mathrm{s}^{-1}$ & $4.55 \pm 1.71$ & $-3.0 \pm 8.4$ & $-8.6 \pm 7.3 * *$ & $-8.8 \pm 9.0 * *$ & $-8.7 \pm 8.8 * *$ \\
\hline FEF75\% L $\cdot \mathrm{s}^{-1}$ & $1.47 \pm 0.89$ & $-5.7 \pm 11.4$ & $-12.6 \pm 6.5 * * *$ & $-11.3 \pm 10.0 * *$ & $-12.6 \pm 11.8 * *$ \\
\hline PEF $L \cdot s^{-1}$ & $8.55 \pm 1.96$ & $-0.9 \pm 6.4$ & $-0.5 \pm 7.7$ & $-1.9 \pm 6.4$ & $-3.3 \pm 6.8$ \\
\hline TL,CO $\mathrm{mmol} \cdot \mathrm{min}^{-1} \cdot \mathrm{kPa}^{-1}$ & $8.64 \pm 1.99$ & $-0.1 \pm 3.8$ & $-2.2 \pm 4.1$ & $-4.4 \pm 5.4 * *$ & $-0.5 \pm 3.3$ \\
\hline$T \mathrm{~L}, \mathrm{CO}$,corr $\mathrm{mmol} \cdot \mathrm{min}^{-1} \cdot \mathrm{kPa}^{-1}$ & $8.46 \pm 2.18$ & $0.3 \pm 5.2$ & $-0.8 \pm 5.7$ & $-3.4 \pm 7.0$ & $0.2 \pm 7.3$ \\
\hline$V \mathrm{~A} \quad \mathrm{~L}$ & $5.70 \pm 1.29$ & $1.2 \pm 1.6$ & $1.2 \pm 1.5$ & $1.5 \pm 1.6$ & $0.1 \pm 1.8$ \\
\hline
\end{tabular}

The mean difference \pm SD from the baseline before HBO treatment is given for each variable. FVC: forced vital capacity; FEV1: forced expiratory volume in one second; FEF25-75\%: mean forced mid-expiratory flow rate; FEF25\%, FEF50\%, FEF75\%: forced expiratory flows at 25, 50 and 75\% of FVC expired; PEF: peak expiratory flow; TL,CO: transfer factor of the lung for carbon monoxide; $T \mathrm{~L}, \mathrm{CO}$, corr: correction in TL,CO to a haemoglobin concentration of $146 \mathrm{~g} \cdot \mathrm{L}^{-1} ; V_{\mathrm{A}}:$ alveolar volume. $* *: \mathrm{p}<0.01 ; * * *: \mathrm{p}<0.001$.

had not returned to pretreatment values. FEV1 and FEF25$75 \%$, were still significantly reduced by $3.5 \pm 3.2 \%(\mathrm{p}<$ $0.01)$ and $10.7 \pm 8.4 \%(p<0.001)$, respectively. The box plots in figure 2 show the median and range of changes in FEV1 and TL,CO. The outliers at different times follow-up are represented by different subjects. Their exclusion from the analysis did not alter the statistical significance of the changes.

There was a significant reduction in TL,CO on day 21 of treatment only and TL,CO was completely normalized 1 month later. After correction for changes in haemoglobin concentration, the change in TL,CO was not significant. There were no changes in $V \mathrm{~A}$ and, thus, the changes in $K C O$ showed the same pattern as the changes in TL,CO.

There was no significant difference between males and females for any changes in the lung function variables. The relative changes in the lung function variables did not correlate with their baseline value or with age.

\section{Discussion}

Recent studies have shown that reductions in small airways conductance and TL,CO may precede changes in vital capacity with continuous oxygen exposure [7, 8] and that the exposure to hyperoxia contributes to the longterm effects of diving on the lung [9]. In this study with intermittent exposure to hyperoxia, which is substantially different from the continuous low-dose exposure during a saturation dive, the same pattern of changes in pulmonary function took place without any changes in vital capacity.

The changes were small, but cannot readily be attributed to differences in the technique of performing a forced expiratory manoeuvre between examinations. With greater effort at the start of the manoeuvre, FEV1 and forced expiratory flow rates can be reduced because of the thoracic gas compression effect [10]. There were, however, no changes in the effort-dependent indices, PEF and FEF25\%, of the flow-volume loop. In addition, forced expiratory flow rates were probably measured at the same absolute lung volume, since there were no changes in FVC or VA, implying that the indices of forced expiratory flow rates at low lung volumes should be directly comparable [5]. An effect of training or learning to perform the forced expiratory manoeuvre would result in a small increase in FVC and FEV1 [11], but this was not seen. By conducting the
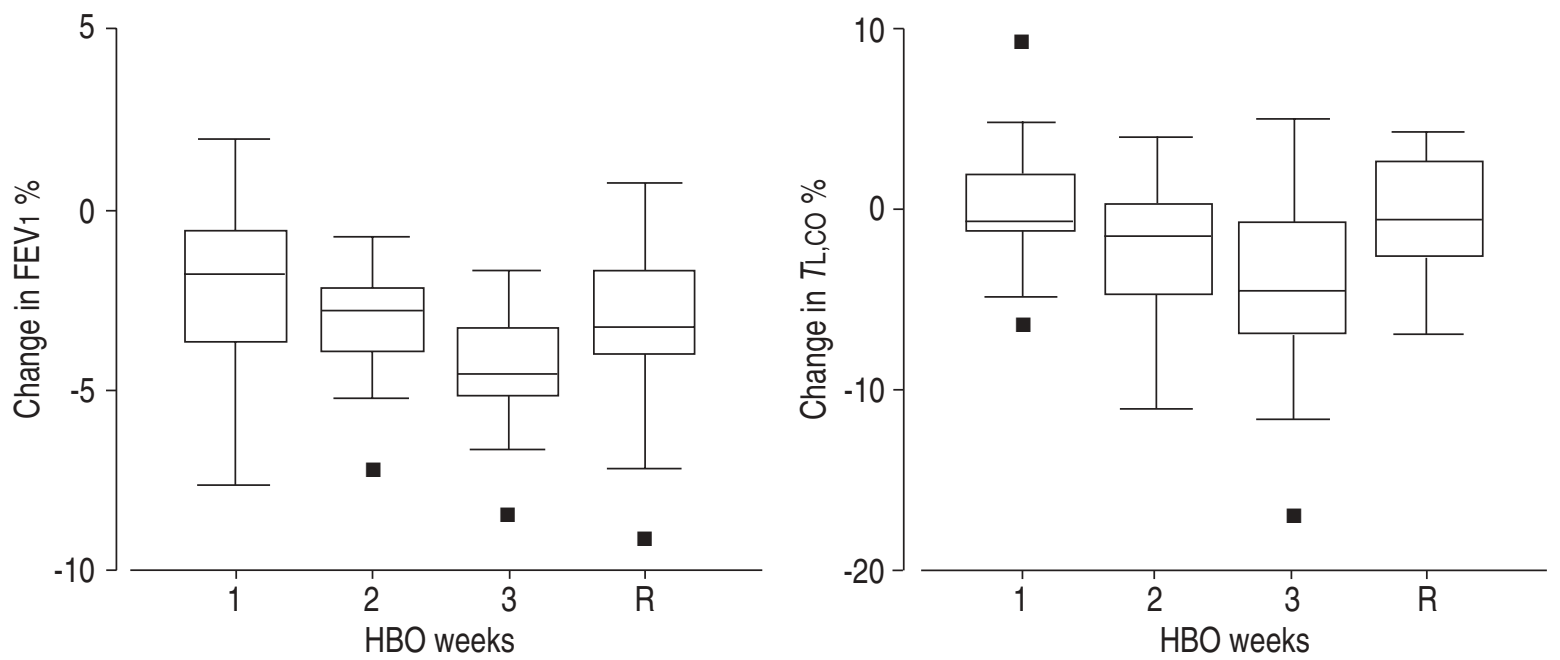

Fig. 2. - Median, 25-75\% percentiles and 5-95\% range of changes in forced expiratory volume in one second (FEV1) and transfer factor of the lung for carbon monoxide (TL,CO) during hyperbaric oxygen (HBO) treatment for 21 days and after 4 weeks' recovery $(\mathrm{R})$. Individual outliers $(\mathbf{\square})$ were represented by different subjects at different follow-up examinations. 
tests strictly according to the procedures of the ERS on the same instrument with the same trained technician, measurement error will be reduced to a minimum.

Pulmonary oxygen toxicity is well characterized with respect to changes in vital capacity in response to continuous exposures to partial pressures of oxygen $>50 \mathrm{kPa}$ [2]. The concept of units pulmonary toxic dose (UPTD) as introduced by CLARK and LAMBERTSEN [2], is widely used for calculating acceptable oxygen exposure under different conditions such as in diving procedures and treatment tables for diving-related disease. Less is known about changes in other lung function variables, in particular in response to exposure to low levels of hyperoxia. In a study with continuous exposure to a $\mathrm{PO}_{2}$ of $40-50 \mathrm{kPa}$ for 28 days, as in a deep saturation dive, where no changes in vital capacity were expected or found, there was a decrease in the forced expiratory flow rates at low lung volumes and in TL,CO. The reduction in the flow rates was linear, as judged from the daily measurements during the exposure [8], and persisted up to 3 yrs after the exposure [9]. There was also a reduction in $\mathrm{TL}, \mathrm{CO}$ of $10 \%$ after correction for changes in haemoglobin concentration which was normalized 1 month after the exposure. In another study, in which no changes in vital capacity were predicted or found, with continuous exposure to a $\mathrm{PO}_{2}$ of $300 \mathrm{kPa}$ for $3.5 \mathrm{~h} \mathrm{[7],}$ there were significant reductions in FEV1, and FEF25-75\% of 5.9 and $11.8 \%$, respectively, and a significant increase in phase III of the single-breath nitrogen washout test $(\delta$ $\mathrm{N}_{2}$ ) A reduction in small airways conductance may thus take place before changes in vital capacity are seen and this study supports these findings.

Exposure to hyperoxia reduces the stimulus for haemoglobin and red blood cell production and, after saturation dives, a reduction in haemoglobin concentration of 5$8 \%$ is usually seen $[8,12]$. The reduction in $T \mathrm{~L}, \mathrm{CO}$ in this study could apparently be explained by the reduction in haemoglobin concentration. This could, however, be a statistical coincidence since the variance in $T \mathrm{~L}, \mathrm{CO}$,corr is larger than in TL,CO because of the additional variance caused by measurement error of haemoglobin concentration. The changes in TL,CO are small however and, as in saturation dives, normalized 1 month after the exposure.

Oxygen toxicity is attenuated when the exposure is intermittent, implying that a cumulative dose, which would have resulted in a reduction in vital capacity if given continuously, can be given intermittently without resulting in changes in vital capacity. This development of tolerance to hyperoxia has also been demonstrated in survival studies of animals exposed to hyperoxia [13] and in cell cultures where there is a stimulation to increased synthesis of antioxidant enzymes [14]. These enzymes are essential in the detoxification of oxygen radicals With the HBO treatment protocols in common use, the daily exposure is equivalent to 275 UPTD, which is not expected to give changes in vital capacity, and the exposure is interrupted by two periods of breathing air for $5 \mathrm{~min}$. The cumulative exposure over approximately 5 days of treatment, 1,425 UPTD, will give a mean reduction in vital capacity of $10 \%$ if given continuously [2]. Over a 3-week HBO treatment period changes in vital capacity are still not seen.

In this study, the reduction in FEV 1 and maximal expiratory flow rates was approximately the same as with continuous exposure to a $\mathrm{PO}_{2}$ of $40-50 \mathrm{kPa}$ for 4 weeks. As for changes in vital capacity with oxygen exposure, the dose-response relationships may be exponential and not linear and there are still too few studies to attempt to model the effect on other lung function variables in healthy subjects. The susceptibility to oxidative stress and induction of defence mechanisms may be very different in healthy subjects than in patients who have this treatment for some underlying disease, which already may stress defence mechanisms.

In conclusion, the reduction in pulmonary function after three weeks of hyperbaric oxygen treatment is not considered to be of any clinical significance unless repeated treatment series are considered or pulmonary function is reduced before treatment. If so, the risk of pulmonary barotrauma has to be considered as a contra-indication for hyperbaric oxygen treatment in addition to the risk of a further reduction in lung function.

\section{References}

1. Thom SR, ed. Hyperbaric Oxygen Therapy: A Committee Report. Bethesda, MD, Undersea and Hyperbaric Medical Society, 1992.

2. Clark JM, Lambertsen CJ. Pulmonary oxygen toxicity: a review. Pharmacol Rev 1971; 23: 37-133.

3. Hendricks PL, Hall DA, Hunter WL Jr, Haley PJ. Extension of pulmonary oxygen tolerance in man at 2 ATA by intermittent oxygen exposure. J Appl Physiol 1977; 42: 593-599.

4. Cotes JE. Allowance for the effect on transfer factor of variation in haemoglobin concentration and in capillary oxygen tension. In: Lung Function. 4th Edn. London, Blackwell Scientific Publications, 1979; pp. 245-246.

5. Quanjer PhH, Tammeling GJ, Cotes JE, Pedersen OF, Peslin R, Yernault J-C. Lung volumes and forced ventilatory flows. Eur Respir J 1993; 6: Suppl. 16, 5-40.

6. Cotes JE, Chinn DJ, Quanjer PhH, Roca J, Yernault J-C. Standardization of the measurement of transfer factor (diffusion capacity) Eur Respir J 1993; 6: Suppl. 16, 41-52.

7. Clark JM, Jackson RM, Lambertsen CJ, Gelfand R, Hiller WDB, Unger M. Pulmonary function in men after oxygen breathing at 3.0 ATA for 35 h. J Appl Physiol 1991; 71: 878-885.

8. Thorsen E, Segadal K, Reed JW, Elliott C, Gulsvik A, Hjelle JO. Contribution of hyperoxia to reduced pulmonary function after deep saturation dives. J Appl Physiol 1993; 75: 657-662.

9. Thorsen E, Kambestad BK. Persistent small airways dysfunction after exposure to hyperoxia. J Appl Physiol 1995; 78: 1421-1424.

10. Krowka MJ, Enright PL, Rodarte JR, Hyatt RE. Effect of effort on measurement of forced expiratory volume in one second. Am Rev Respir Dis 1987; 136: 829-833.

11. Burrows B, Lebowitz MD, Camilli AE, Knudson RJ. Longitudinal changes in forced expiratory volume in one second in adults. Methodologic considerations and findings in healthy nonsmokers. Am Rev Respir Dis 1986; 133: 974-980.

12. Thorsen E, Segadal K, Myrseth E, Påsche A, Gulsvik A. Pulmonary mechanical function and diffusion capacity after deep saturation dives. Br J Ind Med 1990; 47: 242-247.

13. Harabin AL, Survanashi SS, Weathersby PK, Hays JR, Homer LD. The modulation of oxygen toxicity by intermittent exposure. Toxicol Appl Pharmacol 1988; 93: 298-311.

14. Harabin AL, Braisted JC, Flynn ET. Response of antioxidant enzymes to intermittent and continuous hyperbaric oxygen. J Appl Physiol 1990; 69: 328-335. 